\title{
RESULTS FROM THREE DIMENSIONAL SPECTRAL CLASSIFICATION OF POPULATION II STARS USING THE DDO PHOTOMETRIC SYSTEM
}

\author{
W. OSBORN* \\ Inst. Venezolano de Astronomia and Univ. de Los Andes, Mérida, Venezuela
}

\begin{abstract}
The David Dunlap Observatory (DDO) intermediate-band photometric system described in the previous paper has been used to observe a number of red giant branch, horizontal branch, and asymptotic branch members in each of the five globular clusters M3, M5, M10, M13, and M92. A calibration of the DDO system is described by which it was possible to determine the effective temperatures, surface gravities, $[\mathrm{Fe} / \mathrm{H}]$ values, and masses of the observed stars. The mean $[\mathrm{Fe} / \mathrm{H}]$ values for the clusters were found to be -1.01 for M3, -0.68 for M5, -1.44 for M10, -1.69 for M13, and -1.96 for M92. Evidence was found that the masses of the horizontal branch and asymptotic branch stars are systematically smaller than those of red giant branch members. Two stars were discovered to have $\mathrm{CN}$ bands that are anomalously strong for Population II objects. The observational results have been compared with the theoretical predictions of two detailed Population II evolutionary tracks and in general the agreement is good.
\end{abstract}

In the preceeding paper of this symposium McClure has described the David Dunlap Observatory intermediate-band photometric system (usually referred to as the 'DDO system') and has shown how the system may be used for three dimensional classification of G and K stars. Several applications of DDO photometry for studies of Population I objects, along with the results to date, were discussed. In this paper it is shown that the DDO system can also be applied successfully to problems involving Population II stars.

Observations with the DDO system have been used to determine effective temperatures, surface gravities, metal abundances, and mass estimates for a number of latetype globular cluster stars. The principle aim of this program was to obtain data suitable for comparison with the predictions of the detailed Population II evolutionary tracks that are being computed by several research groups, for example, Iben and Rood (1970) and Demarque and Mengel (1971a, b). The amount of observational data presently available for comparison with such calculations is small.

Selected for observation in the program were stars in each of the globular clusters M3, M5, M10, M13, and M92. These clusters are all bright, well-studied objects that were accessable to the available telescope and cover a range of metallicities from extremely metal poor to moderately metal rich. Within each cluster the stars were selected on the basis of four criteria: (1) the star is located within ten minutes of arc of the cluster center, (2) the star has a position in the broad-band cluster HR diagram appropriate for a red giant branch, red horizontal branch, or asympotic branch member, (3) the star is brighter than $V=16$, and (4) on deep plates of the cluster the star has at least $20^{\prime \prime}$ in separation from any other star. The first two of these criteria gave the

* Visiting student, Kitt Peak National Observatory, which is operated by the Association of Universities for Research in Astronomy, under contract with the National Science Foundation. 
star a high probability of being a late-type physical member of the cluster while the last two criteria insured that DDO colors could be obtained that would be of sufficient accuracy to yield reliable data and free from contamination from background stars.

DDO photometry was obtained for a total of thirty-three stars in the five clusters. From their positions in the cluster HR diagrams, sixteen of the stars are members of the red giant branches, four are red horizontal branch members, four are asymptotic branch members, and six stars were classified as probable upper asymptotic branch members but could possibly be instead red giants. Three of the stars were shown by the DDO photometry to be foreground dwarfs. The observations were obtained with the 84 -in. telescope (213-cm aperture) of the Kitt Peak National Observatory equipt with an integrating photometer and utilizing a twelve seconds of arc diaphragm. As well as the four basic filters of the DDO system - Filters 48, 45, 42, and 41, the characteristics and uses of which were given in the paper by McClure - the two supplementary ultraviolet filters of the system - Filters 38 and 35 , which have peak wavelengths of 3813 and $3458 \AA$ and half-widths of 331 and $370 \AA$ respectively - were also observed. The latter two filters are used to form the color indices $C(38-42)$, a measure of the metallic line-blanketing, and $C(35-38)$, a measure of the Balmer discontinuity. These two additional indices are necessary for unambigous three dimensional classification of Population II stars because of the loss of sensitivity of the $C(41-42)$ index when the $\mathrm{CN}$ bands become weak.

To obtain the desired physical parameters of the globular cluster stars from their observed DDO colors, use was made of previously derived calibrations of the system

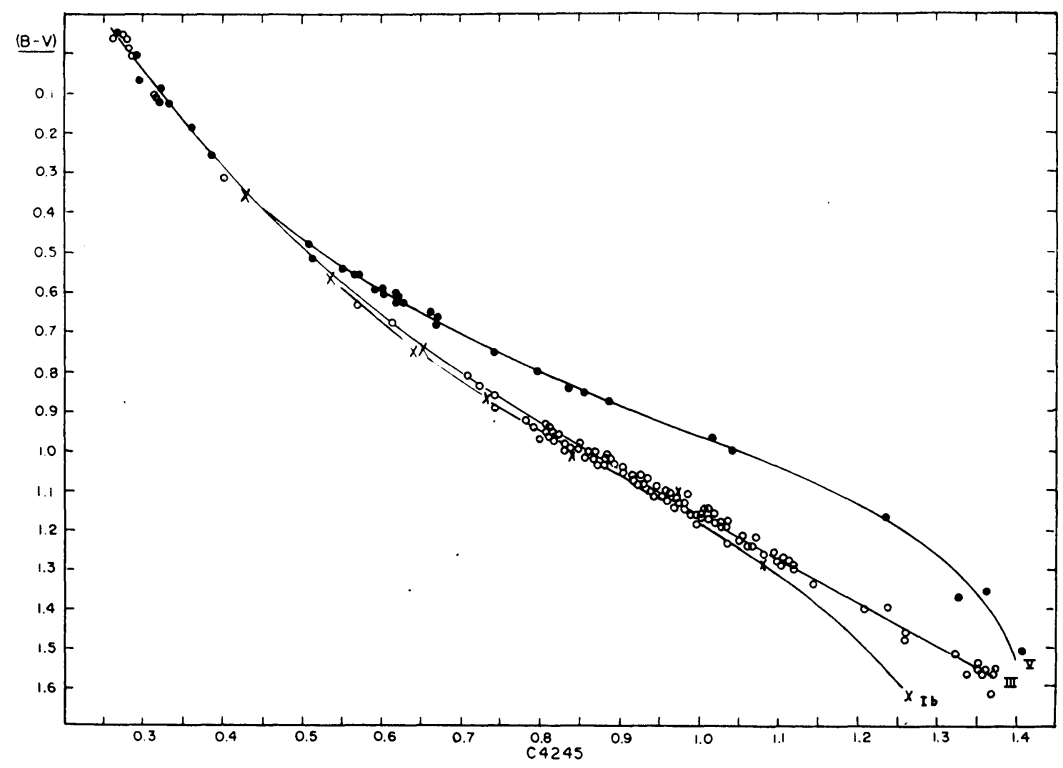

Fig. 1. A plot of $B-V$ vs the DDO temperature index $C(42-45)$. Only stars known to be of MK luminosity class Ib, III, or V and with normal $U B V$ colors (i.e. no ultraviolet excess or deficiency) are plotted. 
with which for the spectral range F5-K5 the DDO colors of a star (either Population I or Population II) can be used to determine its effective temperature, its surface gravity, and its metal abundance. The details of these calibrations and their derivations have been presented elsewhere (Osborn, 1971) but a brief summary will be given.

The effective temperature and surface gravity calibrations were based on previous calibrations of these quantities with $(B-V)$ and MK spectral type. The correlations adopted are given in Table I. Thus, for each case the determination of the relationship between either $(B-V)$ or MK spectral class and the appropriate DDO color index, which could be easily found from the over 500 stars for which all three types of information are available, led directly to the calibration. This process is illustrated in Figures 1 and 2 . The first figure shows the relation between $(B-V)$ and the DDO temperature indicator $C(42-45)$ and the second figure the temperature calibration that results using Table I. Figures 3-5 show in a similar manner the derivation of the surface gravity calibration.

The metal abundance calibrations were obtained directly without recourse to previous calibrations. An exhaustive search of the literature was made for F5-K5 stars with published iron to hydrogen abundance ratios. The search yielded a list of 303 determinations for 231 stars, the data from which were placed on a self-consistent

\section{TABLE I}

Relation adopted between MK spectral type, $(B-V)$, effective temperature, and surface gravity

\begin{tabular}{llllllllll} 
Sp. & $(B-V)$ & & \multicolumn{5}{c}{ Eff. temperature } & \multicolumn{3}{c}{ Log gravity } \\
& Ib & III & V & Ib & III & V & Ib & III & V \\
\hline F5 & 0.35 & 0.43 & 0.44 & 6630 & 6510 & 6550 & 1.7 & & 4.4 \\
F8 & 0.57 & 0.54 & 0.52 & 6020 & 6010 & 6120 & 1.7 & & 4.4 \\
G0 & 0.75 & 0.65 & 0.58 & 5670 & 5760 & 5880 & 1.6 & 3.4 & 4.4 \\
G2 & 0.87 & 0.75 & 0.62 & 5540 & 5430 & 5720 & 1.5 & 3.3 & 4.4 \\
G5 & 1.01 & 0.86 & 0.68 & 5000 & 5180 & 5590 & 1.3 & 3.1 & 4.5 \\
G8 & 1.11 & 0.93 & 0.74 & 4830 & 4940 & 5470 & 1.2 & 2.8 & 4.5 \\
K0 & 1.19 & 1.02 & 0.81 & 4580 & 4720 & 5240 & 1.1 & 2.5 & 4.5 \\
K1 & 1.27 & 1.09 & 0.86 & 4400 & 4530 & & 1.0 & 2.3 & 4.5 \\
K2 & 1.34 & 1.17 & 0.91 & 4260 & 4350 & 4850 & 0.9 & 2.1 & 4.5 \\
K3 & 1.46 & 1.26 & 0.96 & 4140 & 4050 & 4660 & 0.8 & 1.9 & 4.5 \\
K4 & 1.54 & 1.40 & 1.01 & 4010 & 3840 & & 0.7 & 1.7 & 4.5 \\
K5 & 1.61 & 1.50 & 1.14 & 3690 & 3610 & 4140 & 0.6 & 1.5 & 4.5
\end{tabular}

system of $[\mathrm{Fe} / \mathrm{H}]$ values and mean values derived for the stars with more than one determination. Then, using the stars on the list for which DDO photometry was available, the calibrations were obtained by plotting the $[\mathrm{Fe} / \mathrm{H}]$ of the star versus its color anomaly, this being defined as the difference between the observed value of the abundance sensitive color index and the average value of the index for stars of similar spectral type and of nearly solar abundance. Note that for Population II stars the DDO system has two abundance indicators: $C(41-42)$ and $C(38-42)$. The two associated 


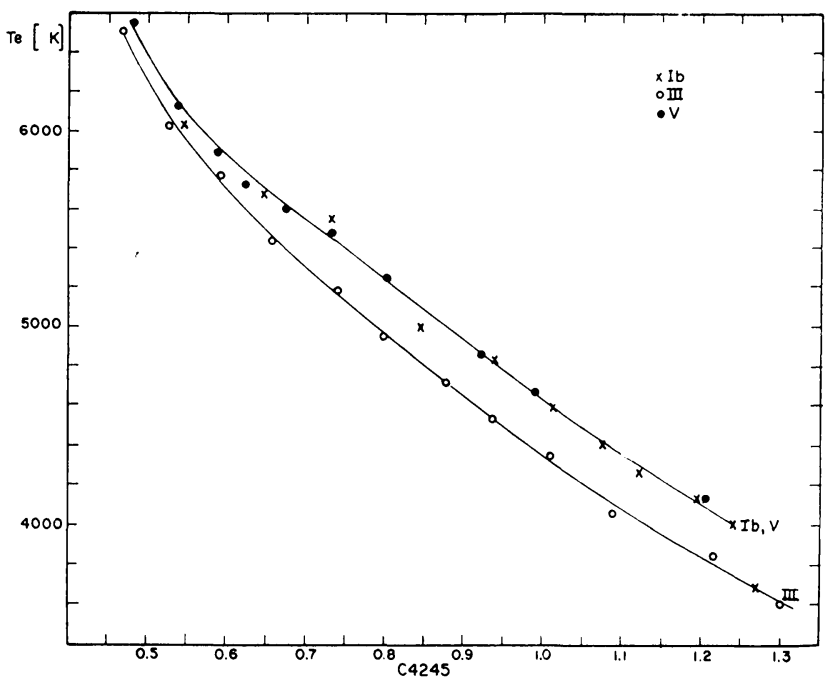

Fig. 2. The relation between $C(42-45)$ and effective temperature that results from the previous figure using the data of Table I.

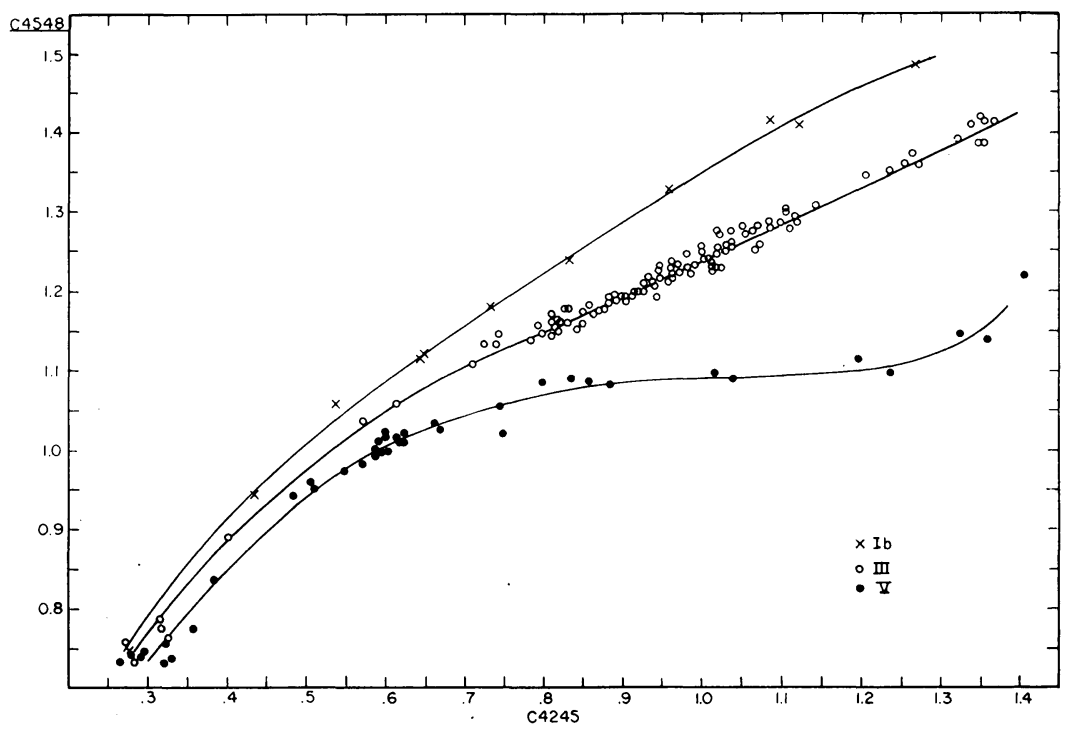

Fig. 3. The $C(45-48)$ vs $C(42-45)$ diagram for luminosity class Ib, III, and V stars.

color anomalies are denoted $\delta C_{m}$ (from $C_{m}$, the gravity corrected from of the $C(41-42)$ index) and $\delta 3842$. The abundance calibration derived from the $C(38-42)$ index is shown in Figure 6.

The derivation of the physical quantities of the program stars proceeded by first adopting apparent visual (AV) distance moduli and values of interstellar reddening 


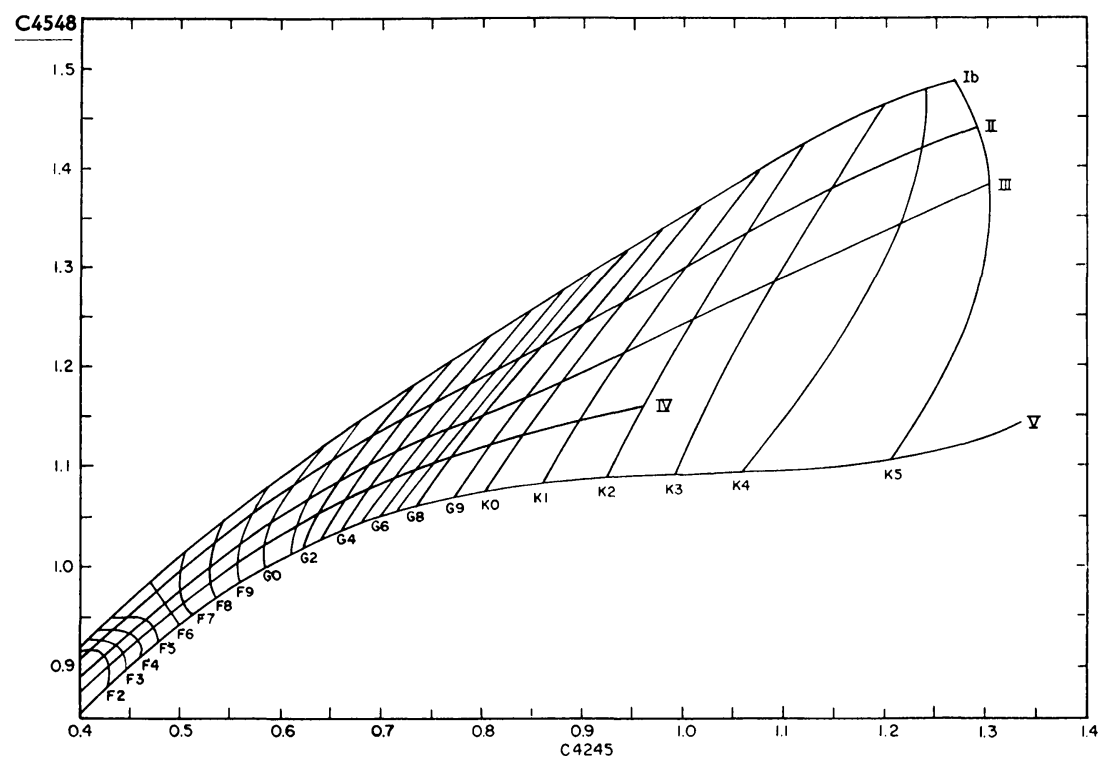

Fig. 4. The relation between MK spectral type and position in the $C(45-48)-C(42-45)$ diagram that was derived using the MK spectral classes of the stars plotted in Figure 3.

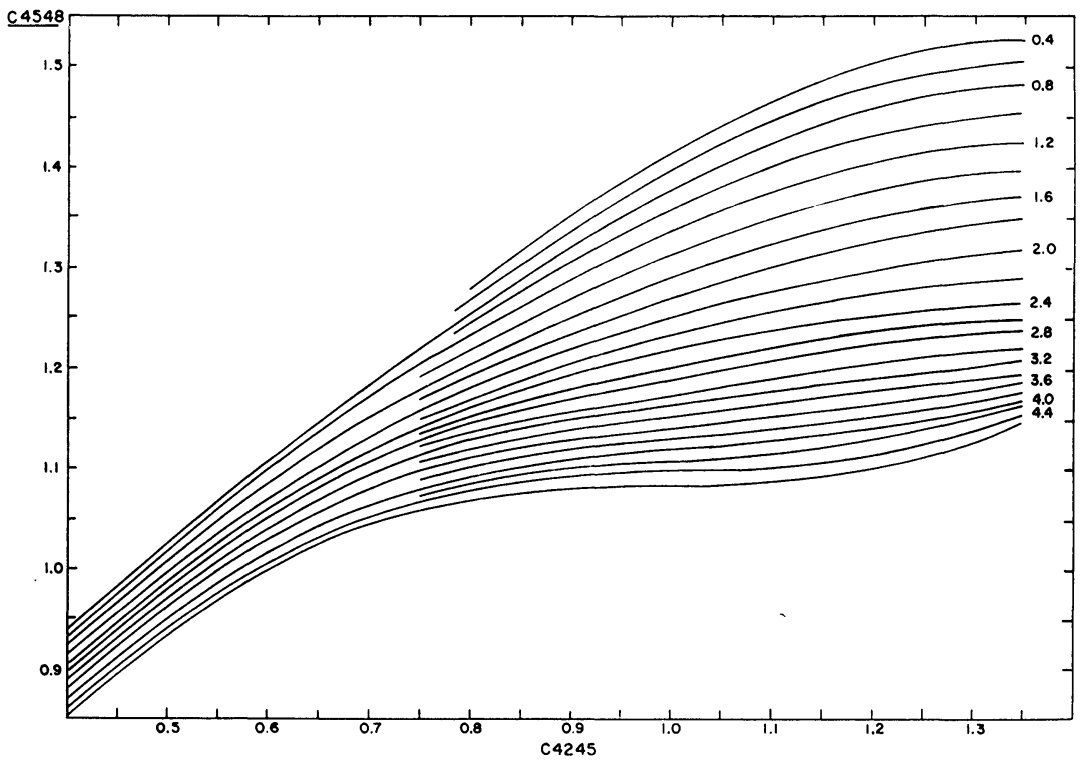

Fig. 5. The lines of constant $\log g$, from $\log g=0.4$ to 4.6 , in the $C(45-48)-C(42-45)$ diagram that were obtained using the surface gravities of Table I and Figure 4. 


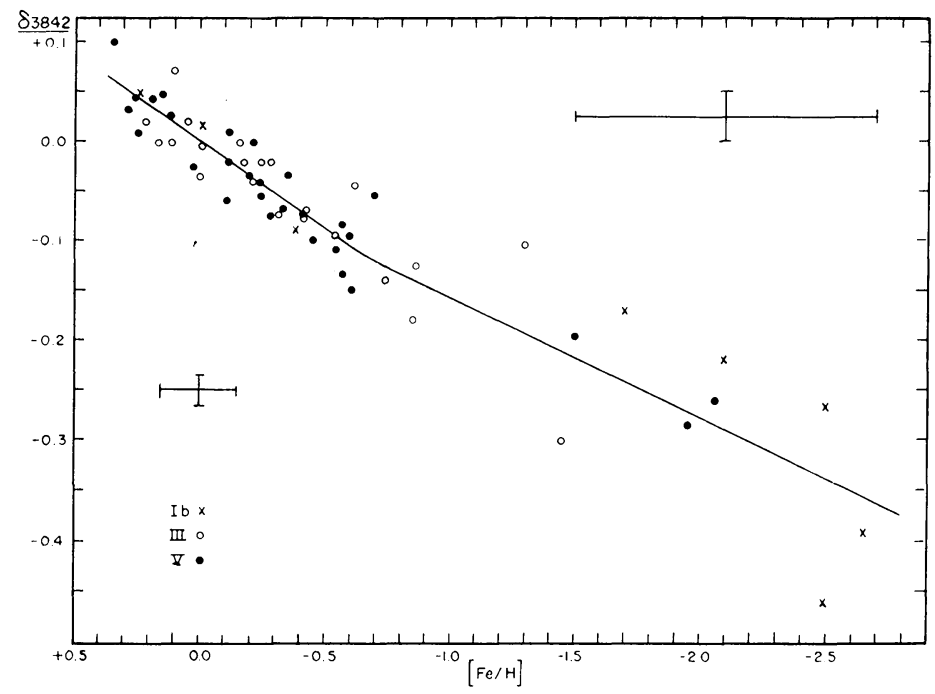

Fig. 6. The correlation between $\delta 3842$ and [Fe/H]. Typical error bars of the points for stars of nearly solar abundance and for extremely metal deficient stars are shown.

for the five clusters (Table II) with which absolute magnitudes were calculated for the stars and their observed DDO colors corrected for reddening. Then, application of the calibrations to the un-reddened colors gave an effective temperature, a surface gravity, and an $[\mathrm{Fe} / \mathrm{H}]$ value for each star. By combining the temperature and gravity results with the absolute magnitude, a mass for the star could also be calculated.

TABLE II

Adopted distance moduli and reddening values

\begin{tabular}{llllll} 
& M3 & M5 & M10 & M13 & M92 \\
\hline$(m-M)_{\mathrm{AV}}$ & 15.3 & 14.8 & 14.4 & 14.4 & 14.5 \\
$E(B-V)$ & 0.005 & 0.035 & 0.26 & 0.015 & 0.02 \\
\hline
\end{tabular}

The principle results from the data are the following. First, mean $[\mathrm{Fe} / \mathrm{H}]$ values have been found for the five clusters, the values being -1.01 for M3, -0.68 for M5, -1.44 for M10, -1.69 for M13, and -1.96 for M92. The uncertainity of each value is of the order of \pm 0.3 , which is approximately a factor of two better than previous results. It is noted that M13 was found to be somewhat more metal poor than usually quoted, in particular significantly more metal poor than M3. The relative ordering of these two clusters has been in doubt for some time.

The second result is that the calculated masses for the stars in the post helium flash stages of evolution, that is the horizontal branch and asymptotic branch stars, were found to be systematically smaller than the masses derived for the pre-helium flash red giants. This systematic trend was found for all five clusters studied and a $t$-test 
for significance on the combined data gave a reliability at the $98 \%$ level. Nevertheless, in view of the small sample sizes and the uncertainities in the individual mass values, this finding stands in need of confirmation. If confirmed it would have important implications in regard to the interpretation of globular cluster HR diagrams using stellar evolution theory.

Third, two stars - one each in M5 and M10 - were discovered to have anomalously strong $\mathrm{CN}$ bands, as measured by the DDO C(41-42) index, in relation to other globular cluster stars. In all but these two cases the globular cluster stars were found to have $C(41-42)$ indices similar to those exhibited by luminosity class IV stars, reflecting the well known fact that their $\mathrm{CN}$ bands are much weaker than what would be expected from their low surface gravities, which are comparable to those of class Ib or class II stars. For the two anomalous stars, however, $C(41-42)$ indicated $\mathrm{CN}$ bands considerably stronger, equivalent to luminosity class III bands. The surface gravities derived for the two stars were similar to those of other globular cluster stars of their temperatures hence the $\mathrm{CN}$ band enhancement does not appear to be a gravity effect. Other stars in globular clusters with enhanced carbon molecule features in their spectra have previously been reported in M13 (Popper, 1947), M92 (Strom and Strom, 1971), and $\omega$ Centauri (Harding, 1962; Stock and Wing, 1971).

Finally, the derived data have been compared with the detailed Population II evolutionary tracks of Demarque and Mengel (1971a, b). The agreement in general is good. The position in the physical HR diagram of the horizontal branch, the slope of the Hayashi line, and the effective temperature separation of the red giant and asymptotic branches are all as predicted. The red giants are found to be somewhat cooler than predicted but this could easily be caused by uncertainities in some of the physics used in the theoretical calculations, notably in the constants employed in the mixing length theory.

The work described in' this paper was performed as part of a $\mathrm{Ph}$. D. dissertation in the Department of Astronomy, Yale University. The observational material was obtained at the Kitt Peak National Observatory in the period January to November 1969.

\section{References}

Demarque, P. and Mengel, J. G.: 1971a, Astrophys. J. 164, 316.

Demarque, P. and Mengel, J. G.: 1971b, Astrophys. J. 164, 469.

Harding, E. A.: 1962, Observatory 82, 205.

Iben, I. and Rood, R. T.: 1970, Astrophys. J. 159, 605.

Osborn, W. H.: 1971, Positions of Globular Cluster Stars in the Physical HR Diagram, Ph. D. Thesis, Yale University

Popper, D. M.: 1947, Astrophys. J. 105, 204.

Stock, J. and Wing, R.: 1972. Bull. Am. Astron. Soc. 4, 324.

Strom, S. E. and Strom, K. M.: 1971, Astron. Astrophys. 14, 111. 